\title{
Further reflections on recent updates to perioperative beta-blocker guidelines
}

\author{
Homer Yang, MD
}

Received: 12 April 2010/Accepted: 16 April 2010/Published online: 6 May 2010

(C) Canadian Anesthesiologists' Society 2010

\section{To the Editor,}

"For every complex problem, there is an answer that is clear, simple, and wrong." H.L. Mencken.

In a recent editorial on perioperative $\beta$-blockers, London $^{1}$ noted that, "The current process (on guidelines for perioperative $\beta$-blockers) appears to be limited increasingly to the...few effective Class I practices..., leaving the majority of real world clinical controversy in the nebulous Class II category." I write to provide several additional perspectives to this discussion. The foremost viewpoint, given the potential problems of guidelines, ${ }^{2}$ is that the issue is not about the guidelines per se, but about the often overlooked postoperative care on surgical wards.

The pathogenesis of perioperative myocardial infarction (PMI) is complex. Coronary artery disease is a major factor in the pathogenesis of PMI, but PMI also occurs in patients without critical stenosis or any identifiable coronary lesions. ${ }^{3}$ Genetic predisposition, postoperative hypercoagulability, and elevated levels of perioperative stress hormones are additional factors. While much is known about preoperative risk factors in predicting PMI, little is known about the manner in which postoperative risk factors influence PMI. This is the reality, in spite of the knowledge that it is postoperative myocardial ischemia rather than pre- and intraoperative ischemia ${ }^{4,5}$ that has been identified as an independent predictor of adverse cardiac outcomes, suggesting that the postoperative period is more important.

Some of the current controversies of the POISE study ${ }^{6}$ centre on the pharmacogenomics and the dose of $\beta$-blockers perioperatively. While CYP2D6 genotypes predict the

H. Yang, MD ( $\square)$

University of Ottawa, Ottawa, ON, Canada

e-mail: hyang@ottawahospital.on.ca plasma concentrations of metoprolol, plasma concentrations do not predict clinical effects of metoprolol. ${ }^{7}$ Pharmacokinetically, metoprolol succinate (extended release) has a greater first pass effect, hence, at least $25 \%$ less bioavailability compared with metoprolol tartrate (immediate release). ${ }^{8}$ Metoprolol succinate $200 \mathrm{mg}$ is equivalent to metoprolol tartrate $65 \mathrm{mg} \mathrm{q} 12 \mathrm{hr} .{ }^{9}$ Furthermore, during the postoperative period in the POISE study, a titration protocol was used in order to avoid "toxicity". Thus, based on current evidence, it may be premature to focus on pharmacogenomics of $\beta$-blockers, and it is potentially misleading to equate dosages of the extended formulation to the immediate release formulation without recognizing the first pass effect.

As alluded to above, the postoperative period may be more clinically important than either the preoperative or the intraoperative period in terms of temporal targeting of interventions to reduce PMI. In this context, one possible interpretation regarding some of the controversy related to the POISE study is that, in addition to the fact that perioperative $\beta$-blockers reduce the incidence of PMI, the study findings exposed an unanticipated interaction between $\beta$-blockers and other postoperative outcomes, including stroke. We recognize that patients being managed postoperatively on surgical wards have a limited number of assessments. Based on this fact, it is possible that a largescale trial, such the POISE study, involving thousands of postoperative evaluations in high-risk patients was able to unmask an interaction between perioperative $\beta$-blocker administration and postoperative risk factors, such as bleeding, anemia, or even sepsis, that were not easily predictable in the preoperative period.

In the current debate, the adverse effects in the POISE study are difficult to reconcile. Many practitioners have suggested that these adverse events could be eliminated or reduced by further fine-tuning preoperative risk factors. If 
one accepts the premise that there are significant postoperative risk factors that cannot be predicted easily in the preoperative period, then one may come to the realization that the risk stratification based on preoperative factors may be reaching its limit. An alternate interpretation is that the current strategies of perioperative $\beta$-blockade are akin to a "shot-gun" approach; hence, one sees "collateral" damage. Knowing that the postoperative period may be more important in predicting PMI, that the pathogenesis of PMI is complex and not necessarily predictable preoperatively, I suggest that more research into the postoperative period would offer an opportunity for more targeted utilization of perioperative $\beta$-blocker therapy, akin to a "rifle" approach, hence, reducing "collateral" damage. For example, while it is not easy to predict preoperatively which patient will bleed excessively, it is possible to focus during the postoperative period on those patients whose bleeding was abnormal. In my view, as noted at the outset, the broader issue of perioperative $\beta$-blocker therapy concerns postoperative care-it is time to shift our research efforts to improve on the delineation of postoperative risk factors and to establish a more targeted utilization of perioperative $\beta$-blockers. The guidelines will follow.

Competing interests Dr. Yang is a principal investigator of the POISE $^{6}$ trial.

\section{References}

1. London MJ. New practice guidelines for perioperative beta blockade from the United States and Europe: incremental progress or a necessary evil? Can J Anesth 2010; 57: 301-12.

2. Kavanagh BP. The GRADE system for rating clinical guidelines. PLoS Med 2009; 6: e1000094.

3. Ellis $S G$, Hertzer NR, Young JR, Brener S. Angiographic correlates of cardiac death and myocardial infarction complicating major nonthoracic vascular surgery. Am J Cardiol 1996; 77: 1126-8.

4. Mangano DT, Browner WS, Hollenberg M, London MJ, Tubau JF, Tateo IM. Association of perioperative myocardial ischemia with cardiac morbidity and mortality in men undergoing noncardiac surgery. The Study of Perioperative Ischemia Research Group. N Engl J Med 1990; 323: 1781-8.

5. Raby KE, Barry J, Creager MA, Cook EF, Weisberg MC, Goldman $L$. Detection and significance of intraoperative and postoperative myocardial ischemia in peripheral vascular surgery. JAMA 1992; 268: 222-7.

6. POISE Study Group; Devereaux PJ, Yang H, Yusuf S, et al. Effects of extended-release metoprolol succinate in patients undergoing non-cardiac surgery (POISE trial): a randomised controlled trial. Lancet 2008; 371: 1839-47.

7. Fux R, Morike K, Prohmer AM, et al. Impact of CYP2D6 genotype on adverse effects during treatment with metoprolol: a prospective clinical study. Clin Pharmacol Ther 2005; 78: 378-87.

8. Gattis WA. Metoprolol CR/XL in the treatment of chronic heart failure. Pharmacotherapy 2001; 21: 604-13.

9. McBride BF, White CM. Critical differences among beta-adrenoreceptor antagonists in myocardial failure: debating the MERIT of COMET. J Clin Pharmacol 2005; 45: 6-24. 